\title{
FORMULATION AND EVALUATION OF MICROSPONGE GEL FOR TOPICAL DELIVERY OF ANTIFUNGAL DRUG
}

\author{
VISHAL YADAV, PRAKASH JADHAV, SHAILAJA DOMBE*, ANJALI BODHE, PRANALI SALUNKHE \\ Arvind Gavali College of Pharmacy, Jaitapur, Satara 415004, India \\ Email: shailajadombe@rediffmail.com
}

Received: 15 Feb 2017, Revised and Accepted: 14 Jun 2017

\begin{abstract}
Objective: The purpose of present study aims to design novel drug delivery system containing oxiconazole nitrate microsponges and to prepare microsponge gel. Oxiconazole nitrate is an antifungal drug used in the treatment of fungal infection having a poor aqueous solubility, side effects and adverse reactions. The microsponge delivery system is unique technology for controlled release of active agents.

Methods: The microsponges were prepared by quasi-emulsion solvent diffusion method by using polymer eudragit S-100 and eudragit L-100. All the formulated microsponges were subjected for various evaluation parameters such as production yield, encapsulation efficiency, particle size analysis and in vitro drug release study. The optimised microsponge formulation F3 and F9 were further formulated as gel formulation for topical delivery. Prepared gel was evaluated for physical parameters like $\mathrm{pH}$, spreadability, viscosity, drug content and in vitro diffusion study and compared with the marketed formulation.

Results: The Fourier transform infrared radiation measurement (FTIR) and Differential scanning colorimetry (DSC) of drug and excipient confirm compatibility. Results revealed that quasi-emulsion solvent diffusion method is a suitable technique for the preparation of microsponges as most of the formulations were discrete and spherical in shape with a good production yield of $61.44 \%$ to $80.45 \%$ and The highest drug release for F3 and F9 formulation was found to be $87.77 \%$ and $83.24 \%$ respectively for the $8 \mathrm{~h}$. The microsponge gel formulation MGI (F3) showed the controlled release of oxiconazole nitrate for $12 \mathrm{~h}$. The drug release data of optimised batch MGI (F3) were fitted into different kinetic models and showed that the drug
\end{abstract} release from gel formulation follows zero order release.

Conclusion: As compared to conventional formulation, the prepared microsponge gel are expected to remain on the skin for a longer time, gradually releasing their contents over the time. Hence, oxiconazole nitrate microsponges and microsponge gel prepared in this study are promising as being more useful than conventional formulation therapy.

Keywords: Oxiconazole nitrate, Microsponge, Antifungal drug, Microsponge gel, Topical delivery

(C) 2017 The Authors. Published by Innovare Academic Sciences Pvt Ltd. This is an open access article under the CC BY license (http://creativecommons.org/licenses/by/4.0/) DOI: http://dx.doi.org/10.22159/ijap.2017v9i4.17760

\section{INTRODUCTION}

The novel drug delivery systems have been increasingly investigated to achieve targeted and controlled release of drugs as many of conventional delivery systems require high concentrations of active agents to be incorporated for effective therapy because of their low efficiency as delivery systems. Microsponges are highly cross-linked, patented, porous, polymeric microspheres that acquire the flexibility to entrap a wide variety of active ingredients that are mostly used for prolonged topical administration and recently for oral administration. Microsponges are designed to deliver a pharmaceutically active ingredient efficiently at minimum dose and also to enhance stability, elegance, flexibility in formulation, reduce side effects and modify drug release profile [1-3].

The microsponge system can prevent excessive accumulation of ingredients within the epidermis and the dermis. These products are typically presented to the consumer in conventional forms like creams, gels or lotions and they contain relatively high concentration of active ingredients. Microsponges are polymeric delivery systems consisting of porous microspheres that can entrap a wide range of active ingredients such as emollients, fragrances, essential oils, sunscreens, and anti-infective, anti-fungal, and anti-inflammatory agents [4].

There have been concerns related to the conventional topical dosage forms such as lotions, creams, ointments and powder in terms of drug diffusion or release from the vehicle and delivery through the skin. Creams and lotions often provide poor bioavailability of the drug because they are rapidly cleared from the skin and poorly release the drug from the base. Non-hydrophilic ointments are oleaginous, greasy and are non-convenient to patients and also medicated powders for the topical application have short residence time on the skin. Gels are semisolid systems in which the movement of the dispersion medium is restricted by interlacing threedimensional network of particles or solvated macromolecules of dispersed phase. The increased viscosity caused by interlacing and consequently internal friction is responsible for the semisolid state. Also, a gel may consist of twisted matted strands often tied together by stronger types of Vander Waals Forces to form crystalline and amorphous regions throughout the system. The use of gel as a delivery system can increase the residence time of drugs on the skin and consequently enhance bioavailability. Gel delivery systems have several advantages such as the ease of administration, non-greasy, patient compliance, high residence time on the skin and better drug release [5]. Oxiconazole inhibits ergosterol biosynthesis, which is required for cytoplasmic membrane integrity of fungi. It acts to destabilize the fungal cytochrome P450 51 enzyme (also known as Lanosterol 14-alpha demethylase) [6]. This is vital in the cell membrane structure of the fungus. Its inhibition leads to cell lysis. Oxiconazole has also been shown inhibition of DNA synthesis and suppresses intracellular concentrations of ATP. Like other imidazole antifungals, Oxiconazole can increase membrane permeability to zinc, augmenting its cytotoxicity.

Oxiconazole nitrate is a potent anti-fungal drug, used in the treatment of fungal infection. Oxiconazole is a cream or lotion applied to the skin in the treatment of tinea corporis, tinea pedis and tinea cruris. Oxiconazole nitrate is BCS-II drug. It has adverse effects include: Burning, itching, blistering, crusting, dryness or flaking of the skin, scaling, severe redness, soreness, swelling and pain in hairy areas with pus at the root of hair and side effects like pruritus, burning, irritation, erythema, stinging and allergic contact dermatitis and folliculitis, fissuring, maceration rash and nodules.

Thus, alternative routes of administration for these drugs with novel drug delivery are being currently investigated. Oxiconazole nitrate microsponge gel have shown significant drug level in deep tissues such as fascia, muscle and synovium after topical application, which is a desirable feature for the relief of local symptoms with low dose, thereby reducing side effects. 
In the present study, the oxiconazole microsponges were prepared using polymers such as eudragit S-100 and eudragit L-100 at different proportions using an individual polymer with the help of quasi-emulsion solvent diffusion method. The microsponge gel formulations were prepared from optimised oxiconazole nitrate microsponges using carbapol 934 with the help of simple dispersion method [6-7].

\section{MATERIALS AND METHODS}

\section{Materials}

Oxiconazole nitrate was procured from Yarrow Chem Products, Mumbai. Eudragit S-100, Eudragit L-100 was obtained from Research-Lab Fine Chem Industries Mumbai. Carbapol-934 and polyvinyl Alcohol was purchased from Loba Chemie Pvt. Ltd., Mumbai and Cosmo chem respectively. All other reagents used were of analytical grade. The microsponges were prepared by the quasiemulsion solvent diffusion method.

\section{Methods}

The microsponges of respective composition, as shown in table 2, were designed using eudragit S-100, eudragit L-100 as a polymer, polyvinyl alcohol as a stabilizer. Batches were designed for a different drug: polymer ratios and for different concentrations of polyvinyl alcohol at stirring speed of $1500 \mathrm{rpm}$.

The microsponges containing oxiconazole nitrate were prepared by a quasi-emulsion solvent diffusion method using eudragit S-100, eudragit L-100 as a polymer. To prepare the inner phase, the polymer is added to ethyl alcohol and the drug is added to methyl alcohol. Polymer solution and drug solution dissolved under ultrasonication at $35{ }^{\circ} \mathrm{C}$. This solution made inner phase. The inner phase was poured into the PVA solution in water (external phase). Following $2 \mathrm{~h}$ stirring at $1500 \mathrm{rpm}$, the mixture is filtered to separate the microsponges. The microsponges are dried in an air heated oven at $40{ }^{\circ} \mathrm{C}$ for $12 \mathrm{~h}$ and weighed to determine production yield (PY) [8].

Table 1: List of instruments

\begin{tabular}{lll}
\hline S. No. & Instruments & Manufacturer \\
\hline 1 & Single Pan Electronic Balance & Shimadzu corp. model(ATX224) \\
2 & Infrared Spectrophotometer & FTIR-Shimadzu \\
3 & Ultrasonicator & Wensar Electronics Pvt. Ltd., Mumbai \\
4 & Mechanical Stirrer & Remi Equipments Ltd., Mumbai \\
5 & Uagnetic Stirrer & Remi Equipments Ltd., Mumbai \\
6 & Differential Scanning Calorimeter & Dynamica, Model: Halo DB-20. \\
7 & Brook Field Viscometer & Mettler-Toledo DSC 821e, Switzerland \\
8 & Diffusion Cell & LVDV-E Brookfield Viscometer, Eng. Lab, USA \\
9 & Digital Potentiometer & Fabricated Apparatus \\
10. & Motic Digital microscope & Equiptronics Model(EQ-601) \\
11. & Hot air oven & B1 Advanced series. \\
12. & Dissolution test apparatus & Biotechnics India. \\
13. & & Electrolab (TDT)-08LUSP Type I \\
\hline
\end{tabular}

Table 2: Composition of oxiconazole nitrate microsponges

\begin{tabular}{|c|c|c|c|c|c|c|c|c|c|c|c|c|}
\hline \multirow[t]{2}{*}{ Ingredients } & \multicolumn{12}{|c|}{ Formulation } \\
\hline & F1 & F2 & F3 & F4 & F5 & F6 & F7 & F8 & F9 & F10 & F11 & F12 \\
\hline Oxiconazolenitrate (mg) & 100 & 100 & 100 & 100 & 100 & 100 & 100 & 100 & 100 & 100 & 100 & 100 \\
\hline Eudragit S-100(mg) & 750 & 750 & 1000 & 1000 & 1250 & 1250 & --- & --- & --- & --- & --- & --- \\
\hline Eudragit L-100(mg) & --- & --- & --- & --- & --- & --- & 750 & 750 & 1000 & 1000 & 1250 & 1250 \\
\hline Ethanol (ml) & 5 & 5 & 5 & 5 & 5 & 5 & 5 & 5 & 5 & 5 & 5 & 5 \\
\hline Methanol (ml) & 5 & 5 & 5 & 5 & 5 & 5 & 5 & 5 & 5 & 5 & 5 & 5 \\
\hline PVA\% $\mathrm{w} / \mathrm{v}$ & 0.5 & 0.75 & 0.5 & 0.75 & 0.5 & 0.75 & 0.5 & 0.75 & 0.5 & 0.75 & 0.5 & 0.75 \\
\hline Water & 100 & 100 & 100 & 100 & 100 & 100 & 100 & 100 & 100 & 100 & 100 & 100 \\
\hline
\end{tabular}

\section{Evaluation of oxiconazole nitrate microsponges}

\section{Determination of production yield}

The production yield of the microsponges was determined by calculating accurately the initial weight of the raw materials and the final weight of the microsponges obtained.

$$
\begin{aligned}
& \% \text { production yield } \\
& =\frac{\text { Practical mass of Microsponge }}{\text { Theorotical mass(polymer }+ \text { Drug })} \text { X100 ....Eqn(1) }
\end{aligned}
$$

\section{Actual drug content and encapsulation efficiency}

A sample of dried microsponges equivalent to $10 \mathrm{mg}$ was taken into mortar and pestle and add little amount of phosphate buffer of $\mathrm{pH}$ 7.4 and allowed to stand for $24 \mathrm{~h}$. Then transfer content into $100 \mathrm{ml}$ volumetric flask and make up volume to $100 \mathrm{ml}$ with phosphate buffer of $\mathrm{pH}$ 7.4. The solution was filtered through Whatman filter paper (No.41). From the resulting solution take $1 \mathrm{ml}$ into $10 \mathrm{ml}$ volumetric flask and then make up volume to $10 \mathrm{ml}$ with phosphate buffer of $\mathrm{pH}$ 7.4. Drug content was determined by UV spectrophotometer (Dynamica, Halo DB-20) at $261 \mathrm{~nm}$.
The drug content and encapsulation efficiency were calculated using the following formula.

$\%$ Encapsulation Efficiency

$$
\begin{gathered}
=\frac{\begin{array}{c}
\text { Actual drug content } \\
\text { in Microsponge }
\end{array}}{\text { Theorotical drug content }} \mathrm{X} 100 \ldots \text {...Eqn(2) } \\
\text { \%Actual drug content }=\frac{\text { Nact }}{\mathrm{Nms}} \mathrm{X} 100 \ldots . \mathrm{Eqn}(3)
\end{gathered}
$$

Where Nact is the actual oxiconazole nitrate content in weighed quantity of microsponges, $\mathrm{Nms}$ is the weighed quantity of powder of microsponges [8-10].

\section{Motic digital microscopy}

For morphology and surface topography, prepared microsponges can be placed on a glass slide at room temperature and then the surface morphology of the microsponges can be studied by Motic Digital Microscopy (B1 advanced series). Motic Digital Microscopy of a fractured microsponge particle can also be taken to illustrate its ultrastructure. 
The morphology of Oxiconazole nitrate microsponge was examined with a Motic Digital Microscopy. The samples were mounted on a glass slide and observed under $10 \mathrm{X}$ object with the magnification power2048 x 1536 [11].

\section{Particle size analysis}

Particle size analysis of prepared microsponges was carried out by using Motic digital microscope particle size analyzer [B1 advanced series]. Microsponges were dispersed on the slide before running the sample in the instrument, to ensure that the light scattering signal, particle size was measured, which is within instrument's sensitivity range [11].

\section{Infrared spectroscopy}

It was determined by Fourier Transform Infrared Spectrophotometer (FTIR-Shimadzu) using KBr pellet method. FTIR spectra of oxiconazole nitrate, physical mixture(s) of oxiconazole nitrate and eudragit S-100, eudragit L-100, carbapol 934 and microsponge formulation were recorded in the wavenumber range of 4000 to 400 $\mathrm{cm}^{-1}[11]$.

\section{Differential scanning calorimetry (DSC)}

Thermal analysis is an important evaluation technique to find any possible interaction between the drug and excipients. Such interaction can be identified by any change in the thermogram. Thermogram of oxiconazole nitrate microsponge formulation was obtained using DSC instrument (PerkinElmer 4000) equipped with an intercooler. Indium standard was used to calibrate the DSC temperature and enthalpy scale. The powder sample of microsponges was hermetically kept in the aluminium pan and heated at a constant rate of $10{ }^{\circ} \mathrm{C} / \mathrm{min}$, over a temperature range of $30^{\circ} \mathrm{C}$ to $300^{\circ} \mathrm{C}$ under a nitrogen atmosphere of the flow rate of 20 $\mathrm{ml} / \mathrm{min}[11]$.

\section{In vitro drug release study}

The dissolution profile of Microsponges can be studied by use of dissolution apparatus USP (Type I) with a modified basket consisted of $5 \mu \mathrm{m}$ stainless steel mesh. A sample equivalent to $100 \mathrm{mg}$ of oxiconazole nitrate was taken in the basket. The speed of the rotation is $100 \mathrm{rpm}$ and temperature of $37 \pm 0.5^{\circ} \mathrm{C}$ was maintained throughout the experiment. The dissolution medium $(900 \mathrm{ml})$ is phosphate buffer $\mathrm{pH} 7.4$ while considering solubility of actives to ensure sink conditions. At fixed intervals, aliquots were withdrawn and replaced with fresh dissolution medium. Samples from the dissolution medium can be analysed by UV spectrophotometer (Dynamica, Halo DB-20) at $261 \mathrm{~nm}$ at various intervals. The concentration of drug released at different time intervals was determined by measuring absorbance [12].

\section{Formulation of microsponges entrapped oxiconazole nitrate gel}

Promising microsponges (formulation prepared by Quasi emulsion solvent method) Formulations of microsponges F3, F9 containing oxiconazole nitrate equivalent to $1 \% \mathrm{w} / \mathrm{w}$ was incorporated into the gel base composed of Carbapol 934 ( $35 \mathrm{mg}$ ), Sodium benzoate $(50 \mathrm{mg})$, Propylene glycol $(0.75 \mathrm{ml})$, Triethanolamine (quantity sufficient) and distilled water up to10 $\mathrm{ml}$ [13].

\section{Evaluation of oxiconazole nitrate gel}

\section{Visual inspection}

The prepared gel formulation of microsponges was inspected visually for their color, texture and appearance [11].

\section{pH measurement}

The $\mathrm{pH}$ of gel formulation was determined by using digital $\mathrm{pH}$ meter. One gram of gel was dissolved in $100 \mathrm{ml}$ distilled water and stored for two hours. The measurement of $\mathrm{pH}$ of the formulation was done [14].

\section{Spreadability studies}

One of the criteria for a gel to meet the ideal qualities is that it should possess good spreadability. It is the term expressed to denote the extent of the area to which gel readily spreads on application to the skin or affected part. The therapeutic efficacy of a formulation also depends upon its spreading value. Spreadability is expressed in terms of time in seconds taken by two slides to slip off from gel placed in between the slides under the direction of certain load. Lesser the time is taken for separation of two slides, better the spreadability.

Spreadability was determined by glass slides and a wooden block, which was provided by a pulley at one end. By this method, spreadability was measured on the basis of Slip and Drag characteristics of gels. A ground glass slide was fixed on this block. An excess of gel (about $1 \mathrm{gm}$ ) of different formulations was placed on the ground slide. The gel was then sandwiched between this slide and another glass slide having the dimension of fixed ground slide. Excess of the gel was scrapped off from the edges. The top plate was then subjected to pull of $20 \mathrm{gms}$, lesser the time is taken for separation of two slides better the spreadability.

Spreadability was then calculated using the following formula:

$$
\mathrm{S}=\mathrm{M} \times \mathrm{L} / \mathrm{T} \ldots . . \text { Eqn }(4)
$$

Where, $\mathrm{S}=$ is the spreadability, $\mathrm{M}=$ is the weight in the pan (tied to the upper slide), $\mathrm{L}=$ is the length moved by the glass slide $\mathrm{T}=$ represents the time taken to separate the slide completely from each other $[11,15]$.

\section{Viscosity measurement}

The viscosity of the different gel formulations was determined using a Brookfield viscometer with spindle no. 64 at $100 \mathrm{rpm}$ at temperature $25^{\circ} \mathrm{C}$. The viscosity of the optimised formulation was determined as such without dilution using Brookfield Viscometer (Model-LVDV-E). Brookfield Viscometer consists of a cup, which is stationary and a spindle which is rotating. Different sized rotating spindles are used and immersed in the test material. For liquids with low viscosity, large size spindles (large diameter and surface area) are used while for higher viscosity liquids small spindles (small diameter and surface area) are used. Rotate the spindle in the microsponge gel till we get a constant dial reading on the display of the viscometer. This procedure is repeated three times for reproducible results [15-16].

\section{Drug content}

1 gm of oxiconazole nitrate microsponge gel was accurately weighed and dissolved using methanol, sonicated for a period of 10-15 min and made up to the mark in $100 \mathrm{ml}$ volumetric flask with methanol. From this $10 \mathrm{ml}$ was pipetted out and diluted to $100 \mathrm{ml}$ with methanol and the final dilution was made using distilled water to get a concentration within Beer's range. The absorbance was measured by UV spectrophotometer (Dynamica, Halo DB-20) at $260 \mathrm{~nm}$ against blank gel treated in the same manner as a sample [15].

\section{In vitro diffusion study}

In vitro studies of the gel were carried out across the egg membrane extracted by using the concentrated $\mathrm{HCl}$. The receptor compartments were filled with phosphate buffered saline (PBS) pH 7.4, Study was carried out using excised egg membrane. Franz diffusion cell with $30 \mathrm{ml}$ receptor compartment and effective area $4.52 \mathrm{~cm}^{2}$ was placed on a thermostatic magnetic stirrer and the temperature was maintained at $37^{\circ} \mathrm{C}$ throughout the study. Selected batches of drug microsponge gel (MGI, MGII, M. F.) were used for the diffusion study using diffusion cell. Aliquots, each of $1 \mathrm{ml}$ volume were withdrawn at specific intervals and replaced by an equal volume of the receptor medium. The aliquots were suitably diluted with the receptor medium. Release studies were carried out over a period of $12 \mathrm{~h}$ at regular intervals. Samples were withdrawn and analyzed by UV spectrophotometer (Dynamica, Halo DB-20) at 261 $\mathrm{nm}[11,15]$.

\section{In vitro drug release kinetic study}

To determine the drug release mechanism and to compare the release profile differences among microsponge gel formulations, the data obtained from the drug released amount and time were used. The drug release kinetics was analyzed with mathematical models like Zero order, First order, Higuchi matrix, Hixson crowell and Korsmeyer-peppas model. Several kinetic models have been 
proposed to describe the release characteristics of a drug from the matrix. The three parameters were used to study the release mechanism i.e. release rate constant (k), correlation coefficient (R), and release exponent (n) and determine the best fit model for optimised formulation [17]. The release data was analyzed with the following mathematical models:

\section{Zero order kinetics}

Drug dissolution from pharmaceutical dosage forms that do not disaggregate and release the drug slowly (assuming that area does not change and no equilibrium conditions are obtained) can be presented by the following equation:

$$
\mathrm{Q}=\mathrm{K}_{0} \mathrm{t} \ldots . . \text { Eqn (4) }
$$

Where $Q$ is the amount of drug released at time $t, K_{0}$ is the zeroorder rate constant expressed in units of concentration/time and $\mathrm{t}$-is the time in hours.

The pharmaceutical dosage forms following this profile, release the same amount of drug by a unit of time. This model represents an ideal release profile in order to achieve the prolonged pharmacological action [17].

\section{First order kinetics}

This model has also been used to describe absorption and/or elimination of some drugs, although it is difficult to conceptualise this mechanism on a theoretical basis.

$$
\begin{gathered}
\mathrm{Q}_{1}=\mathrm{Q}_{0} \mathrm{e}^{-\mathrm{K}_{1} \mathrm{t}} \text { or } \\
\log \mathrm{Q}_{1}=\log \mathrm{Q}_{0}+\frac{\mathrm{K} 1 \mathrm{t}}{2.303} \ldots . \text { Eqn (5) }
\end{gathered}
$$

Where Q1 is the amount of drug released in time $t$,

$Q_{0}$ is the initial amount of drug in the solution and

$\mathrm{K}_{1}$ is the first order release constant.

The pharmaceutical dosage form following this dissolution profile, such as water soluble drugs in porous matrices releases the drug in such a way that is proportional to the amount of drug remaining in its interior, in such a way that the amount of drug released by a unit of time diminishes [17].

\section{Higuchi matrix model}

This model is used to study the release of water soluble and low soluble drugs incorporated in semisolid and/or solid matrices. Mathematical expressions were obtained for drug particles dispersed in a uniform matrix behaving as the diffusion media. It describes drug release as a diffusion process based on the Fick's law, square root time dependent [17].

$$
\mathrm{Q}=\mathrm{K}_{\mathrm{H}} \mathrm{t}^{1 / 2} \ldots . \text { Eqn }(6)
$$

Where $Q$ is the amount of drug release in time $t$,

$\mathrm{K}_{\mathrm{H}}$ is the Higuchi dissolution constant.

\section{Korsmeyer-peppas model}

Korsmeyer developed a simple, empirical model, relating exponentially the drug release to the elapsed time $(t)$.

$$
f \mathrm{t}=\mathrm{a} \cdot \mathrm{t}^{\mathrm{n}} \ldots . \text { Eqn (7) }
$$

Where $\mathrm{a}$ is a constant incorporating structural and geometric characteristics of the drug dosage form, $\mathrm{n}$ is the release exponent, indicative of the drug release mechanism and function of $\mathrm{t}$ is $\mathrm{Mt} / \mathrm{M} \infty$ (fractional release of drug) [17].

\section{Hixson-Crowell model}

Hixson and Crowell (1931) recognised that the particles regular area is proportional to the cube root of its volume. They derived the equation:

$$
\mathrm{W}_{0}{ }^{1 / 3}-\mathrm{W}_{\mathrm{t}}{ }^{1 / 3}=\kappa \mathrm{t} . . . \text { Eqn (8) }
$$

Where $W_{0}$ is the initial amount of drug in the pharmaceutical dosage form, $\mathrm{Wt}$ is the remaining amount of drug in the pharmaceutical dosage form at time $t$ and $\kappa$ (kappa) is a constant incorporating the surface volume relation. The equation describes the release from systems where there is a change in surface area and diameter of particles or tablets. To study the release kinetics, data obtained from in vitro drug release studies were plotted as the cube root of drug percentage remaining in matrix versus time [17].

\section{RESULTS AND DISCUSSION}

Determination of production yield and encapsulation efficiency

The production yield of all batches was ranged from $61.44 \%$ to $80.45 \%$. It was found that production yield was greatly affected by drug: polymer ratio as well as by concentration of polyvinyl alcohol. It was indicated that increasing polymer concentration, increased production yield while increasing polyvinyl alcohol concentration, decreased production yield.

Use of the higher amounts of PVA while preparing microsponges at a higher drug: polymer ratios caused slightly an increased viscosity of the dispersed phase. When solvents in inner phase were diffused out, nearly all of the dispersed phase was converted to the form of solid microsponges and separated particles appeared. The highest drug loading efficiency of these formulations could be explained through the fact that the amount of polymer to per unit drug was greater than that in other formulations.

Table 3: Evaluation of oxiconazole nitrate microsponges

\begin{tabular}{llll}
\hline Batches & Production yield (\%) & Actual drug content (\%) & Encapsulation efficiency (\%) \\
\hline F1 & $63.53 \pm 0.01$ & 60.21 & 60.21 \\
F2 & $61.44 \pm 0.02$ & 54.23 & 54.23 \\
F3 & $77.81 \pm 0.01$ & 79.25 & 79.25 \\
F4 & $73.84 \pm 0.02$ & 75.29 & 75.29 \\
F5 & $80.45 \pm 0.03$ & 80.12 & 80.12 \\
F6 & $78.51 \pm 0.01$ & 78.36 & 78.36 \\
F7 & $64.47 \pm 0.01$ & 58.24 & 58.24 \\
F8 & $63.80 \pm 0.02$ & 53.48 & 53.48 \\
F9 & $76.81 \pm 0.01$ & 77.80 & 77.80 \\
F10 & $75.93 \pm 0.01$ & 75.24 & 75.24 \\
F11 & $79.86 \pm 0.01$ & 79.14 & 79.14 \\
F12 & $78.81 \pm 0.01$ & 76.53 & 76.53 \\
\hline
\end{tabular}

(mean \pm SD; $\mathrm{n}=3$ )

\section{Motic digital microscopy}

The morphology of the microsponges prepared by quasi-emulsion solvent diffusion method and entrapment method was investigated by Motic Digital microscope (B1 Advanced series). The representative motic microscopic image of the microsponges are shown in fig. 1a. The image showed the microsponges to be porous and was having predominantly spherical shape and not much entire.

Oxiconazole nitrate crystals were observed visually. 
The pores were induced by the diffusion of the solvent from the surface of the microsponges. The appearance of the particles was such that they were termed as microsponges. From fig. 1a it was revealed that the characteristic internal structure was a spherical cavity enclosed by a rigid shell constructed from drug and polymer. The inner structure consisted of void spaces. From fig., 1b microsponge gel image by Motic Digital microscope (B1 Advanced series) showed that microsponge are entrapped uniformly in three-dimensional cross-linked networks within the liquid.

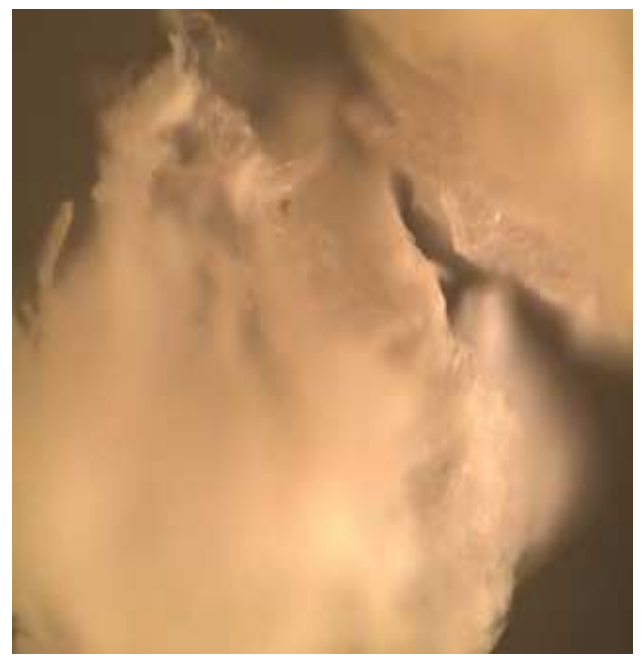

Fig. 1a): Image of oxiconazole nitrate microsponges

\section{Particle size analysis}

The mean particle size of microsponge formulations should be in the range of 5-300 um. The F3 and F9 batch possessed more percent of intact, uniform, spherical particles in optical microscopy; so this batches was chosen for further analysis using Motic Digital Microscope (B1 Advanced series). The F3 and F9 batch results revealed particle size corresponding to $59.7 \mu$ mand $70.7 \mu \mathrm{m}$.

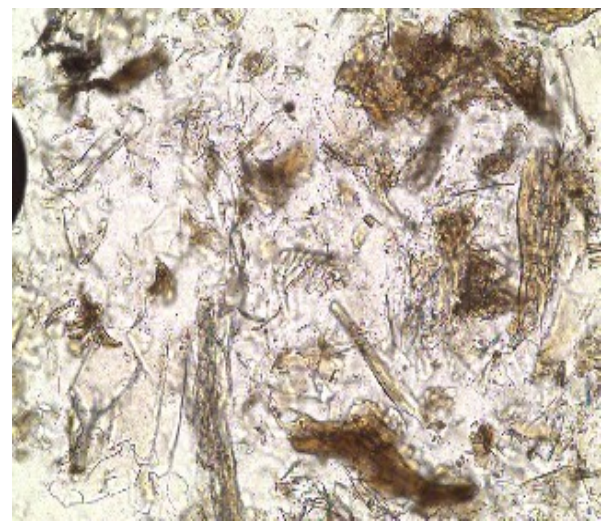

Fig. 1b): Image of oxiconazole nitrate microsponge gel

\section{In vitro drug release study}

The cumulative percent drug release (\% CDR) for all formulations was calculated out. The cumulative percent drug release of all formulations was as quoted in table 4 and 5 .

Table 4: In vitro drug release profile of F1-F6

\begin{tabular}{|c|c|c|c|c|c|c|}
\hline \multirow[t]{2}{*}{ Time (h) } & \multicolumn{6}{|c|}{ Formulations (\% CDR) } \\
\hline & F1 & F2 & F3 & F4 & F5 & F6 \\
\hline 0 & 0 & 0 & $\mathbf{0}$ & 0 & 0 & 0 \\
\hline 1 & 1.76 & 1.30 & 4.44 & 2.84 & 1.85 & 1.32 \\
\hline 2 & 4.22 & 5.86 & 12.71 & 9.79 & 7.40 & 6.18 \\
\hline 3 & 13.15 & 11.10 & 20.55 & 10.80 & 15.26 & 14.04 \\
\hline 4 & 17.70 & 15.63 & 26.64 & 15.36 & 18.50 & 19.95 \\
\hline 5 & 29.16 & 22.02 & 37.56 & 28.77 & 23.74 & 32.05 \\
\hline 6 & 36.53 & 33.95 & 43.57 & 40.15 & 36.19 & 41.50 \\
\hline 7 & 48.72 & 40.52 & 62.81 & 53.31 & 58.23 & 57.55 \\
\hline 8 & 65.68 & 54.58 & 87.77 & 75.65 & 70.66 & 67.71 \\
\hline
\end{tabular}

Table 5: In vitro drug release profile of F7-F12

\begin{tabular}{|c|c|c|c|c|c|c|}
\hline \multirow[t]{2}{*}{ Time (h) } & \multicolumn{6}{|c|}{ Formulations (\% CDR) } \\
\hline & F7 & F8 & F9 & F10 & F11 & F12 \\
\hline 0 & 0 & 0 & $\mathbf{0}$ & 0 & 0 & 0 \\
\hline 1 & 2.13 & 1.94 & 3.45 & 1.85 & 4.72 & 5.14 \\
\hline 2 & 9.44 & 8.33 & 8.22 & 9.44 & 12.09 & 8.19 \\
\hline 3 & 18.50 & 15.33 & 15.63 & 18.50 & 23.24 & 15.99 \\
\hline 4 & 21.92 & 18.13 & 28.09 & 19.80 & 31.07 & 23.15 \\
\hline 5 & 29.60 & 24.70 & 45.72 & 25.44 & 38.05 & 32.28 \\
\hline 6 & 35.52 & 32.56 & 68.81 & 37.65 & 42.12 & 44.19 \\
\hline 7 & 49.03 & 49.58 & 78.53 & 54.76 & 54.66 & 52.33 \\
\hline 8 & 62.62 & 59.57 & 83.24 & 68.64 & 70.14 & 68.62 \\
\hline
\end{tabular}

The drug release was found to be decreased in the range of $87.77 \%$ to $54.58 \%$ for eudragit S-100 as the drug: polymer ratio was changed. The drug release was found to be decreased in the range of $83.24 \%$ to $59.57 \%$ for eudragit L-100 as the drug: polymer ratio was changed. The reason behind is a drug: polymer ratio was increased, the amount of polymer available per microsponge to encapsulate the drug becomes more, thus increasing the thickness of the polymer matrix wall which might lead to longer diffusion path and ultimately to decreased drug release. The highest drug release i.e. $87.77 \%$ was found for the formulation F3, while the lowest 54.58 $\%$ for F2 for eudragit S-100. The highest drug release i.e. $83.24 \%$ was found for the formulation F9, while the lowest $59.57 \%$ for F8 for eudragit L-100. It has been reported that with increasing amount of PVA from batches F1-F12, the drug release went on decreasing. It 
might be due to fact that the polymer matrix releases drug after complete swelling and time required for swelling of the polymer is directly proportional to stabilizer concentration. Batch F3 and F9 showed $87.77 \%$ and $83.24 \%$ drug release in $8 \mathrm{~h}$. Thus they were further formulated as gel formulation as MGI and MGII.

\section{Drug-excipient interaction study by FTIR spectrum:}

FTIR spectroscopic study revealed that there was no appearance of any new peak and disappearance of existing peaks, which indicated that there was no chemical interaction between the drug and polymer used. The IR spectrum exhibited distinctive peaks at $1479.40\left(\mathrm{~cm}^{-1}\right)$ due to $\mathrm{C}=\mathrm{N}$ stretching. The peaks at $1712.35\left(\mathrm{~cm}^{-1}\right)$ is due to the $\mathrm{C}=\mathrm{C}$ stretching and at $752.24\left(\mathrm{~cm}^{-1}\right)$ is due to the $\mathrm{C}-\mathrm{Cl}$ stretching. The peak at 2951.09 is due to the $\mathrm{C}-\mathrm{H}$ methylene stretching. The peak at 1384.89 is due to $\mathrm{N}-\mathrm{O}$ stretching. All the characteristic peaks of oxiconazole nitrate were observed in the IR spectra of physical mixture and oxiconazole nitrate loaded microsponges during the investigation of compatibility study.

Hence IR spectroscopy results showed that the drug was compatible with selected polymer and excipients. This suggested that oxiconazole nitrate was compatible with selected polymers and it was apparently stable in the microsponges.

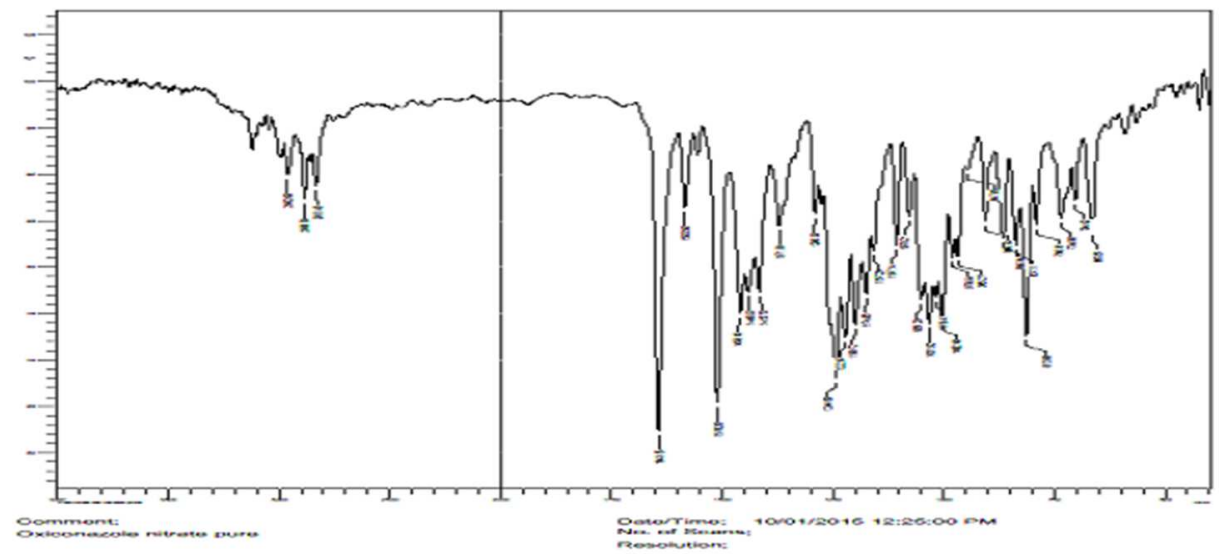

Fig. 2: FTIR spectrum of oxiconazole nitrate

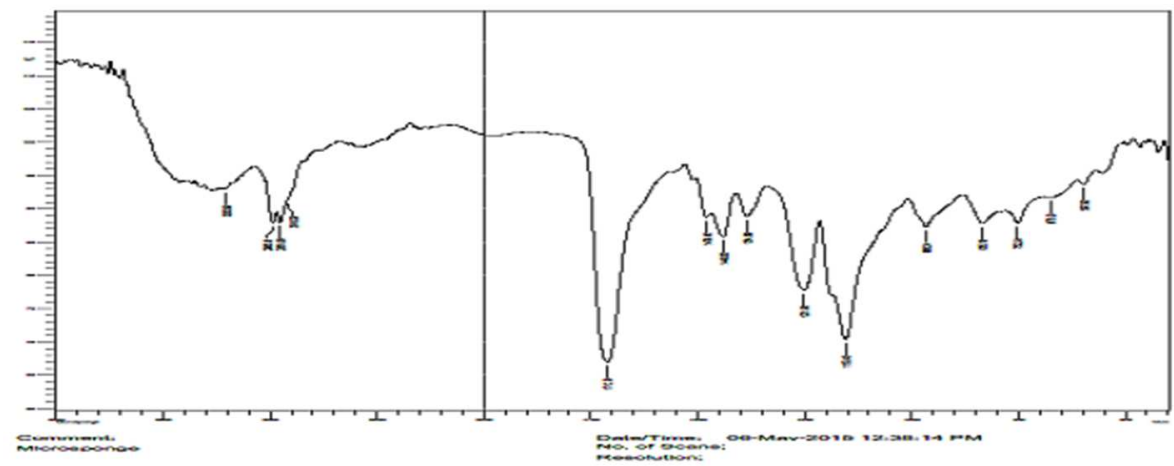

Fig. 3: FTIR spectrum of optimized formulation

\section{Drug-excipient interaction study by DSC thermogram}

In DSC studies, dispersed in polymer showed the same thermal behaviour as a pure compound. In the thermogram, the endothermic peak was observed at $106.31{ }^{\circ} \mathrm{C}$ which does not correspond to the melting point of the pure drug. During formulation of microsponges, the drug was entrapped inside the microsponges and was not available for showing any exothermic peak. Hence, no endothermic peak near to the melting point of the drug was observed confirming the entrapment of drug in microsponges. This indicates that the physical properties of oxiconazole nitrate are altered during formulation of microsponges using eudragit S-100.

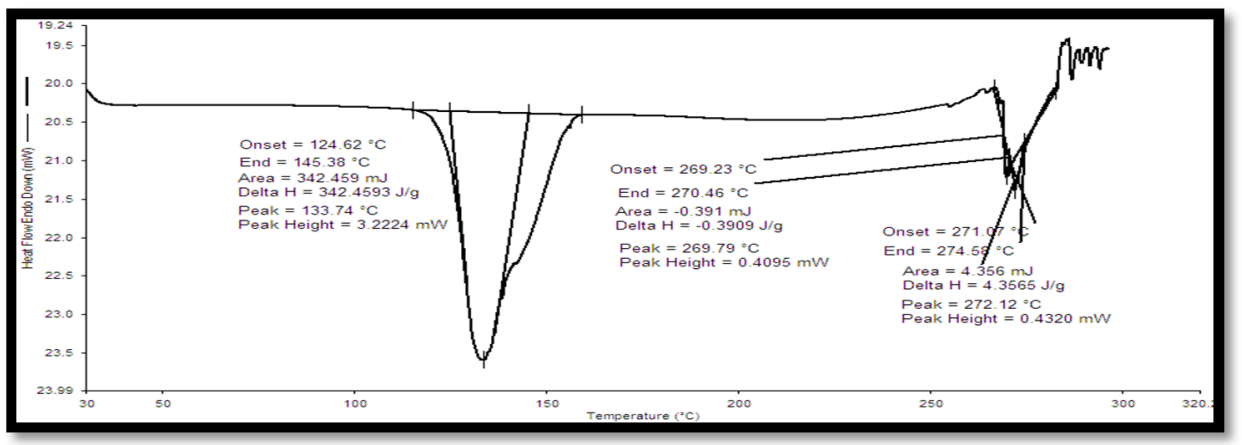

Fig. 4: DSC thermogram of oxiconazole nitrate 


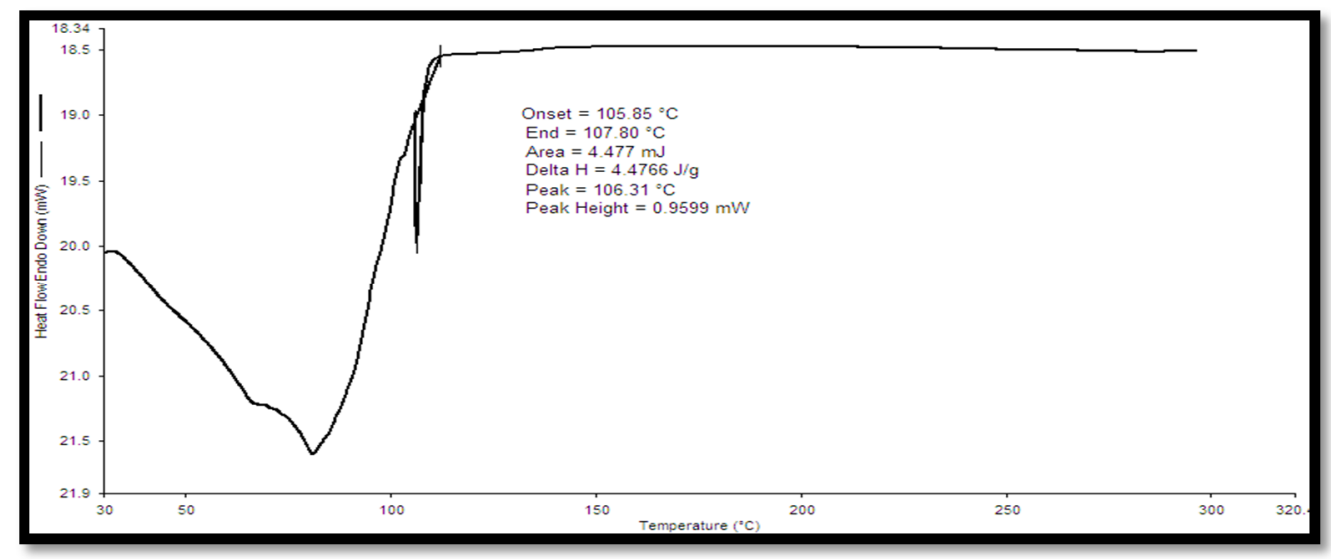

Fig. 5: DSC thermogram of optimized formulation

\section{Oxiconazole nitrate microsponge gel}

\section{Evaluation of oxiconazole nitrate microsponge gel}

\section{Visual inspection}

The prepared gel formulations of oxiconazole nitrate microsponges were inspected visually for their color, texture and appearance. All prepared formulations were pearl white, viscous preparations with a smooth texture and showed good homogeneity with the absence of any lumps and syneresis.

\section{pH measurement}

The $\mathrm{pH}$ value of all prepared formulations was found to be in the range of 6.7 to 6.8 , which was considered to be acceptable to avoid the risk of irritation upon application to the skin.

Table 6: Evaluation of oxiconazole nitrate microsponge gel

\begin{tabular}{llll}
\hline Formulation & PH $^{\mathrm{H}}$ & Spreadability (g. cm/sec) & Viscosity (cps) \\
\hline MGI & $6.8 \pm 0.06$ & $7.5 \pm 0.33$ & $1000 \pm 2$ \\
MGII & $6.7 \pm 0.06$ & $5.0 \pm 0.25$ & $1010 \pm 2$ \\
Marketed formulation & $6.8 \pm 0.06$ & $6.0 \pm 0.17$ & $1025 \pm 0.58$ \\
\hline
\end{tabular}

$($ mean $\pm S D ; n=3)$

\section{Spreadability study}

The values of spreadability indicated that the gel was easily spreadable by a small amount of shear. Spreadability of microsponge gel (MGI) was found to be $7.5 \mathrm{~g}$. $\mathrm{Cm} / \mathrm{sec}$; indicating that spreadability of drug loaded microsponge gel was good.

\section{Viscosity studies}

The viscosities studies for microsponge formulations were carried out. The viscosities of all formulations are shown in Table 6. From above result, microsponge gel (MGI) has required viscosity.

\section{Drug content studies}

Drug content studies for microsponge formulations were carried out. Drug content of all formulations are shown in table 6. The drug content of the formulations showed that the drug was uniformly distributed in the gels. From above result, microsponge gel (MGI) has higher drug content.

\section{In vitro diffusion study}

The in vitro diffusion studies were carried out for all formulations using PBS ( $\mathrm{pH}$ 7.4). In vitro diffusion of formulation MGI, MGII, M. F. is shown in table 7.

Table 7: Amount of drug diffused per unit area of microsponge gel formulations

\begin{tabular}{llll}
\hline \multirow{2}{*}{ Time (h) } & \multicolumn{3}{l}{ Formulations $\mathbf{~ ( \% C A D D / \mathbf { c m } ^ { 2 } \text { ) }}$} \\
\cline { 2 - 4 } & MGI & MGII & Marketed formulation \\
\hline 0 & 0 & 0 & 0 \\
1 & 10.41 & 0.95 & 2.98 \\
2 & 15.64 & 5.73 & 12.65 \\
6 & 19.30 & 11.42 & 27.87 \\
4 & 28.82 & 21.06 & 47.22 \\
5 & 37.92 & 30.42 & 71.17 \\
6 & 46.74 & 39.65 & 80.12 \\
7 & 56.31 & 48.99 & 95.98 \\
8 & 64.83 & 67.99 & ---- \\
9 & 70.21 & 82.53 & ---- \\
10 & 76.64 & 93.53 & ---- \\
11 & 81.82 & ----- & ---- \\
12 & 92.40 & --- & \\
\hline
\end{tabular}


It was observed that the formulation F3 (MGI) showed a higher amount of drug diffused at the end of $12 \mathrm{~h}$ while that of F9 (MGII) and Marketed Formulation showed the higher amount of drug diffused at the end of $10 \mathrm{~h}$ and $7 \mathrm{~h}$ respectively. The results indicated that the $\mathrm{Q}$ value (cumulative amount of drug permeated per unit skin surface area) was controlled for $12 \mathrm{~h}$ at MGI drug: polymer ratio and at $0.5 \%$ $\mathrm{w} / \mathrm{v}$ PVA. The cumulative amount of drug permeated per unit skin surface area (Q) from the microsponge loaded gel formulation MGI was $92.40 \%$. Hence, the microsponge loaded gel formulation MGI was optimized formulation and drug release kinetic study was done.

\section{Drug release kinetic study of optimized formulation MGI (F3)}

To determine the kinetics of release, drug diffusion data were treated with different kinetic equations. Obtained drug diffusion data was fitted to Zero order, First order, Higuchi matrix model, Hixson crowell model and Korsmeyer-peppas model. The three parameters were used to study the release mechanism i.e. release rate constant $(\mathrm{k})$, correlation coefficient $(\mathrm{R})$, release exponent $(\mathrm{n})$. Correlation coefficient $(\mathrm{R})$, release rate constant $(\mathrm{k})$, of optimized formulation F3 (MGI), is reported in table 8.

Table 8: Release kinetics of optimized formulation (F3) MGI

\begin{tabular}{lll}
\hline Model & & Formulation code \\
\hline Zero order & $\mathrm{k}$ & 0.1171 \\
& $\mathrm{R}$ & 0.9969 \\
First order & $\mathrm{k}$ & -0.0020 \\
& $\mathrm{R}$ & 0.9666 \\
Higuchi matrix & $\mathrm{k}$ & 2.5742 \\
& $\mathrm{R}$ & 0.9321 \\
Hixson crowell & $\mathrm{k}$ & -0.0005 \\
& $\mathrm{R}$ & 0.9860 \\
Korsmeyer peppas & $\mathrm{k}$ & 0.1983 \\
& $\mathrm{R}$ & 0.9905 \\
Best fit model & $\mathrm{n}$ & 0.9134 \\
\hline
\end{tabular}

The model that fits the release data was selected based on the correlation coefficient (R) value in various models. The model that gave the high ' $R$ ' value was considered as the best fit of release data. Korsmeyer peppas model best described the sustained release of optimized F3 (MGI) formulation and the diffusion exponent (n) value was found to be 0.9134 suggesting that the Ficks law of diffusion was not followed. From the result, the best fit model for optimised formulation F3 (MGI) is Zero order.

\section{CONCLUSION}

The gel containing microsponges showed the viscous modulus. This study concluded that microsponges prepared with eudragit S-100 and eudragit L-100 in the 1:10 (F3) were more efficient to give extended drug release which released the $87.77 \%$ and $83.24 \%$ drug at the end of 8 h. Microsponge formulation F3 and F9 showed good physical parameter study and was used for formulating into gel, incorporated in the carbapol. The microsponge gel prepared with eudragit S-100 in the batch MGI (F3) was showed good physical parameters and more efficient to give extended drug release which released the $92.40 \%$ drug at the end of $12 \mathrm{~h}$. The optimised formulation MGI (F3) followed Zero order kinetics and non-fiction diffusion. As compared to conventional formulation, these microsponge gel are expected to remain on the skin for a longer time, gradually releasing their contents over the time. Hence, oxiconazole nitrate microsponges and microsponge gel prepared in this study are promising as being more useful than conventional formulation therapy.

\section{CONFLICT OF INTERESTS}

Declared none

\section{REFERENCES}

1. Karthika R, Elango K, Ramesh Kumar K, Rahul K. Formulation and evaluation of lornoxicam microsponge tablets for the treatment of arthritis. Int J Res Article Pharm Innovations 2013;3:29-40.

2. Mehta M, Panchal A, Shah VH, Upadhyay U. Formulation and in vitro evaluation of controlled release microsponge gel for topical delivery of clotrimazole. Int J Adv Pharm 2012;2:93-101.

3. Kaundal A, Bhatia R, Sharma A, Sukrial P. A review on microsponges drug delivery system. Int J Adv Pharm 2014;4:177-81.

4. Pandey P, Jain V, Mahajan SC. A review: microsponge drug delivery system. Int J Biopharm 2013;4:225-30.
5. Sharma S, Pawar S, Jain UK. Development and evaluation of topical gel of curcumin from different combination of polymers formulation and evaluation of herbal gel. Int J Pharm Pharm Sci 2012;4:452-6.

6. https://www.drugbank.ca/drugs/DB00239. [Last accessed on 10 Jan 2017]

7. Maryadele JO' Neil. The Merck Index, An encyclopedia of Chemicals, Drugs and Biological. 14 ${ }^{\text {th }}$ Edition; 2006. p. 6937.

8. Makwana R, Patel H, Patel V. Photostability enhancement of miconazole nitrate by microsponge formulation. Int J Curr Trends Pharm Res 2014;2:437-58.

9. Durgapal S, Mukhopadhyay S, Goswami L. Preparation, characterization and evaluation of floating microparticles of ciprofloxacin. Int J Appl Pharm 2017;9:1-8.

10. Jadhav N, Patel V, Mungekar S, Bhamare G, Karpe M, Kadams V. Microsponge delivery system: an updated review, current status and future prospects. J Sci Ind Res 2013;2:1097-110.

11. Riyaz Ali M Osmani, Aloorkar NH, Ingale DJ, Kulkarni PK, Umme Hani, Bhosale RR, et al. Microsponge based novel drug delivery system for augmented arthritis therapy. Saudi Pharm J 2015;23:562-72.

12. P Yadav, S Nanda. Development and evaluation of some microsponge loaded medicated topical formulations of acyclovir. Int J Pharm Sci Res 2014;5:1395-410.

13. NS Abdelmalak, SF El-Menshawe. A new topical fluconazole microsponge loaded hydrogel: preparation and characterization. Int J Pharm Pharm Sci 2012;4:460-9.

14. Katkade M, Kalkotwar R, Jain N, Patil P, Gadakh R, Naikwade J. Ethyl cellulose based microsponge delivery system for antifungal vaginal gels of tioconazole. J Drug Delivery Ther 2013;3:14-20.

15. R Ravi, SK Senthil Kumar, S Parthiban. Formulation and evaluation of the microsponge gel for an anti acne agent for the treatment of acne. Int J Pharm Sci Res 2013;3:32-8.

16. More HN, Hajare AA. Practical Physical Pharmacy, Career publications. $2^{\text {nd }}$ edition; 2015. p. 153-5.

17. Paulo Costa, Jose Manuel Sousa Lobo. Modeling and comparison of dissolution profiles. Eur J Pharm Sci 2001;13:123-33.

\section{How to cite this article}

- Vishal Yadav, Prakash Jadhav, Shailaja Dombe, Anjali Bodhe, Pranali Salunkhe. Formulation and evaluation of microsponge gel for topical delivery of the antifungal drug. Int J Appl Pharm 2017;9(4):30-37. 